\title{
Microencapsulation of inorganic nanocrystals into PLGA microsphere vaccines enables their intracellular localization in dendritic cells by electron and fluorescence microscopy
}

\author{
Christopher Schliehe ${ }^{a}$, Constanze Schliehe ${ }^{b}$, Marc Thiry $^{b}$, Ulrich I. Tromsdorf ${ }^{b}$, Joachim Hentschel ${ }^{c}$, \\ Horst Weller ${ }^{\mathrm{b}}$, Marcus Groettrup ${ }^{\mathrm{a}, \mathrm{d}, *}$ \\ a Division of Immunology, Department of Biology, Constance University, 78457 Konstanz, Germany \\ b Institute of Physical Chemistry, Hamburg University, 20146 Hamburg, Germany \\ cElectron Microscopy Facility, Department of Biology, Constance University, 78457 Konstanz, Germany \\ d Biotechnology Institute Thurgau at the University of Constance, 8280 Kreuzlingen, Switzerland
}

Keywords:

Antigenicity

Biodegradation

Immunostimulation

Microencapsulation

Microsphere

Polylactic acid

\begin{abstract}
A B S T R A C T
Biodegradable poly-(D,L-lactide-co-glycolide) microspheres (PLGA-MS) are approved as a drug delivery system in humans and represent a promising antigen delivery device for immunotherapy against cancer. Immune responses following PLGA-MS vaccination require cross-presentation of encapsulated antigen by professional antigen presenting cells (APCs). While the potential of PLGA-MS as vaccine formulations is well established, the intracellular pathway of cross-presentation following phagocytosis of PLGA-MS is still under debate. A part of the controversy stems from the difficulty in unambiguously identifying PLGA-MS within cells. Here we show a novel strategy for the efficient encapsulation of inorganic nanocrystals (NCs) into PLGAMS as a tool to study their intracellular localization. We microencapsulated NCs as an electron dense marker to study the intracellular localization of PLGA-MS by transmission electron microscopy (TEM) and as fluorescent labels for confocal laser scanning microscopy. Using this method, we found PLGA-MS to be rapidly taken up by dendritic cells and macrophages. Co-localization with the lysosomal marker LAMP1 showed a lysosomal storage of PLGA-MS for over two days after uptake, long after the initiation of cross-presentation had occurred. Our data argue against an escape of PLGA-MS from the endosome as has previously been suggested as a mechanism to facilitate cross-presentation of PLGA-MS encapsulated antigen.
\end{abstract}

\section{Introduction}

Poly(lactide-co-glycolide) microspheres (PLGA-MS) are spherical, biodegradable polymer particles that can be loaded with a great variety of therapeutic molecules $[1,2]$. They are approved by the US Food and Drug Administration (FDA) to be used as drug delivery system in humans. Recent studies highlight the promising role of PLGA-MS as antigen delivery system for the induction of $\mathrm{CD}^{+}{ }^{+} \mathrm{T}$-cell dependent immune responses against cancer $[3,4]$. They show ideal properties for phagocytotic uptake by professional antigen presenting cells (APCs), like dendritic cells (DCs), and macrophages (MФs), and offer the possibility to co-encapsulate antigenic material with immune stimulatory adjuvants [4].

In this study, we combined the biocompatibility of PLGA-MS with the exceptional properties of inorganic nanocrystals (NCs). NCs are

\footnotetext{
* Corresponding author. Constance University, Chair of Immunology, Universitaetstrasse 10, D-78457 Konstanz, Germany. Tel.: + 497531 882130; fax: + 497531 883102.

E-mail address: Marcus.Groettrup@uni-konstanz.de (M. Groettrup).
}

colloidal particles, which exhibit a size in the low nanometer range. NCs composed of a wide range of inorganic materials can be prepared. Within the nanometer scale, their characteristic properties often differ dramatically from those of the corresponding bulk material. The particle size in turn is responsible for the exceptional change of magnetic, electronic, and optic properties of the NCs. Semiconducting NCs (quantum dots, QDs) show fluorescence at an emission wavelength determined by the intrinsic electronic band gap structure of the material and confinement effects depending on the particle size [5]. Parameters like high photostability, the narrow emission signal and small stokes shift of QDs provide an extraordinary potential for replacing organic dyes within the biological diagnostic [6]. Further examples to illustrate the special properties of NCs are superparamagnetic iron oxide nanoparticles (SPIONs). While the macroscopic iron oxide is ferromagnetic, its corresponding NCs show superparamagnetic characteristics [7]. Among others, the "hot injection" method is a one pot procedure for synthesizing colloidal, monodisperse, and crystalline nanoparticles [8].

A common way to produce PLGA-MS is the so called spray drying evaporation technique [9]. A homogenous emulsion, containing the 
PLGA polymer precursor resomer (organic phase) and usually hydrophilic proteins or other therapeutics (aqueous phase), is sprayed into vacuum. During this process the solvent evaporates and PLGA polymer formation occurs, generating spherical microparticles. Depending on the resomer and the spray drying conditions, the particular properties of microparticles can vary [2]. Using this technique, we introduce a straightforward protocol to efficiently encapsulate NCs of different materials into PLGA-MS. This novel strategy allows the application of the diverse properties of NCs for investigating PLGA-MS function and offers interesting options for the development of laboratory tools or novel strategies to fight cancers.

Three examples are shown to demonstrate the potential of NCs encapsulation. Although PLGA-MS are used as a drug delivery system for years and are intensively studied as a tool to induce T-cell responses, the intracellular fate of microparticles after uptake by APCs is still poorly understood and controversially debated [1014]. Transmission electron microscopy (TEM) studies analysing intracellular distribution of PLGA-MS were so far dependent on osmium tetroxide incorporation [11], colloidal gold labelling [1], or the abnegation of any electron dense marker $[2,13,15,16]$. Here we show that lead sulfide ( $\mathrm{PbS}$ ) QDs serve as an excellent tool to label PLGA-MS with an electron dense marker for replacing toxic osmium tetroxide or rather inefficient colloidal gold labelling. In a second approach we encapsulated fluorescent cadmium selenide (CdSe) QDs to demonstrate their ideal properties for bioimaging. Numerous studies performed microscopy analysis of PLGA-MS labelled with fluorescent dyes like fluorescein isothiocyanate (FITC) or coumarin $[14,16,17]$. However the high photo sensitivity of such dyes hampers to take high resolution images by confocal laser scanning microscopy (LSM), Z-stack analysis, or time course experiments requiring multiple excitation of the same object. Using CdSe QDs encapsulated PLGA-MS, we were able to perform high resolution LSM images of PLGA-MS after phagocytosis by APCs. Using the lysosomal marker LAMP1 we used LSM to show colocalisation with fluorescent PLGA-MS, indicating the lysosomal storage of phagocytosed particles.

Cell sorting based on magnetic beads is a well established and commercially available method to purify individual cell populations from biological samples $[18,19]$. This technique is usually based on covalent labelling of magnetic beads with antibodies specific for a certain lineage marker. Antibody binding attaches magnetic beads to the cell surface and allows purification of the labelled cell type via a magnetic column. In a third experimental setting, we tried to combine the ideal properties of PLGA-MS for phagocytosis with the superparamagnetic characteristics of FeS nanoparticles, to generate magnetic PLGA-MS for a novel cell sorting approach. Rapid internalisation of PLGA-MS by phagocytes was exploited to deplete such cells from a heterogeneous cell suspension. Our results demonstrate that many powerful properties of NCs can be utilized for biomedical research by encapsulation into PLGA-MS.

\section{Materials and methods}

\subsection{Synthesis of quantum dots}

Nanoparticles were synthesized by hot injection method. PbSnanoparticles (PbS org) were prepared as described elsewhere [20], using a TOP/OA stabilizing mixture. The synthesized PbS-nanoparticles were transferred into water by a ligand exchange (PbS aq.). To this aim, particles were incubated with an excess of hydroxyl amine for $30 \mathrm{~min}$. The mixture was centrifuged and the precipitated particles were solved in water. $\mathrm{CdSe} / \mathrm{CdS} / \mathrm{ZnS}$ quantum dots were prepared in a one pot hot injection synthesis as described elsewhere [21,22], using a TOP/TOPO/HDA stabilizing mixture.

\subsection{Poly(lactic-co-glycolic)acid microsphere preparation}

PLGA-MS were prepared from resomer RG502H (Boehringer Ingelheim, Germany) by spray drying as described elsewhere $[3,4]$. Briefly, phosphate buffered saline only (MS-empty) or $50 \mathrm{mg}$ ovalbumin (Grade V, Sigma) in phosphate buffered saline (MS-OVA) (aqueous phase) were emulsified with 5\% PLGA in dichloromethane (organic phase) by ultrasonication (Hielscher, UP200 H, Ampl. 40\%). The emulsion was spray-dried (Mini-Spray-Dryer B-290, Büchi) at a flow rate of $2 \mathrm{ml} / \mathrm{min}$ and inlet/outlet temperatures of $40 / 38^{\circ} \mathrm{C}$. Immediately before use, MS were dispersed in media by ultrasonication. $\mathrm{PbS}$ nanoparticles were encapsulated by either adding particles to the aqueous ( $\mathrm{PbS}$ aq.) or the organic phase ( $\mathrm{PbS}$ org.) of the emulsion. The encapsulation of fluorescent cadmium selenide and magnetic iron oxide nanoparticles was achieved by adding particles to the organic phase of the emulsion.

\subsection{Transmission electron microscopy (TEM)}

Microspheres were embedded in a low-viscosity epoxy resin (Spurr) as described elsewhere [23]. Slices of $100 \mathrm{~nm}$ were prepared and analysed without further contrast agents. BMDCs were grown on petriPERM ${ }^{\circledR}$ dishes (Sigma-Aldrich). Cells were pulsed with PbS quantum dot-labelled PLGA-MS for $1 \mathrm{~h}$. For TEM preparation, cells were fixed after indicated times with $2 \%$ glutardialdehyde solution. Samples were washed in sodium cacodylate buffer and treated with $1 \% \mathrm{OsO}_{4}$. Cells were dehydrated by increasing concentrations of ethanol. After reaching $100 \%$ ethanol, cells were embedded in Spurr. Slices of $80 \mathrm{~nm}$ thickness were prepared and samples further contrasted using lead citrate and uranyl acetate. Images were acquired at $80 \mathrm{kV}$ using a Zeiss EM10-S electron microscope.

\subsection{Cell lines and media}

All cell culture media were purchased from Gibco, Invitrogen. The murine dendritic cell line DC2.4 $\left(\mathrm{H}-2^{\mathrm{b}}\right)$ was a kind gift from K. Rock (University of Massachusetts Medical School Worcester, MA) and cultured in RPMI 1640, 10\% FCS, $100 \mathrm{U} / \mathrm{ml}$ penicillin/streptomycin $(\mathrm{P} / \mathrm{S})$. Bone marrow-derived dendritic cells (BMDCs) were prepared and maintained as described elsewhere [4]. Murine peritoneal macrophages $\left(\mathrm{H}-2^{\mathrm{b}}\right)$ were cultured in RPMI 1640 supplemented with $10 \% \mathrm{FCS}, 100 \mathrm{U} / \mathrm{ml} \mathrm{P} / \mathrm{S}$. The $\mathrm{CD}^{+}{ }^{+}$-cell hybridoma cell line $\mathrm{B} 3 \mathrm{Z}$, specific for the SIINFEKL $\left(\right.$ Ova $\left._{257-264} / \mathrm{K}^{\mathrm{b}}\right)$ peptide of ovalbumin was a kind gift from N. Shastri (University of California, Berkeley, USA) and cultured in IMDM, $10 \%$ FCS, $100 \mathrm{U} / \mathrm{ml} \mathrm{P/S} \mathrm{[24].} \mathrm{The} \mathrm{murine} \mathrm{fibroblast}$ cell line B8 $\left(\mathrm{H}-2^{\mathrm{d}}\right)$ [25] was cultured in IMDM, 10\% FCS, $100 \mathrm{U} / \mathrm{ml} \mathrm{P/S}$.

\subsection{Preparation of primary cells}

Bone marrow-derived dendritic cells (BMDCs) were prepared from naive C57BL/6 mice as previously described [4]. For microscopy BMDCs were differentiated on cover slips and used on day 6. Peritoneal macrophages ( $\mathrm{pM} \Phi \mathrm{s}$ ) were prepared by intra peritoneal (i.p.) injection of $3 \%$ thioglycolate solution into $\mathrm{C} 57 \mathrm{BL} / 6$ mice. After three days, peritoneal cells were washed out of the abdominal cavity. Cells were cultured for 2 days and adherent cells were used for microscopy as well as cross-presentation assays.

\subsection{Confocal laser scanning microscopy (LSM)}

Cells were cultured on cover slips and CdSe quantum dot-labelled microspheres were added on day 2. Unbound microspheres were removed by washing cells with phosphate buffered saline. Cells were fixed in $4 \%$ paraformaldehyde, treated with ammonium chloride solution (50 $\mathrm{mM}$ in phosphate buffered saline), and permeabilized in $0.2 \%$ triton $\mathrm{X}-100$. Samples were blocked with $0.2 \%$ fish gelatine 
(G-7765, Sigma) in phosphate buffered saline. Anti-LAMP1 primary antibody (553792, BD Pharmingen) was diluted $1: 50$ in $0.2 \%$ fish gelatine. Secondary Alexa488 conjugated anti-rat Ig (A11006, Invitrogen) was diluted 1:300 in phosphate buffered saline. Cells were embedded using Dapi-Fluoromount-G (SouthernBiotech). Samples were analysed using an LSM510meta (Zeiss). Cadmium selenide specific fluorescence was excited by UV laser. Microscopy images were processed using the software AxioVs40 V4.7.2.0 (Zeiss).

\subsection{Detection of antigen presentation by LacZ T-cell hybridoma assay}

Presentation of $\mathrm{Ova}_{257-264}$ was detected using the $\mathrm{CD}^{+} \mathrm{T}$-cell hybridoma cell line B3Z [24]. Cells were co-incubated with APCs and $25 \mu \mathrm{g} /$ well of either microspheres containing ovalbumin (MS-OVA) or empty microspheres (MS-empty). After 18 h, LacZ-buffer [0.13\% NP40, $9 \mathrm{mM} \mathrm{MgCl}, 0.15 \mathrm{mM}$ chlorophenolred- $\beta$-D-galactopyranoside (CPRG) (Roche, Germany) in phosphate buffered saline] was added for $4 \mathrm{~h}$ at $37^{\circ} \mathrm{C}$. Absorbance was measured at 570/620 nm using a SpectroFluorPlus spectrometer (Tecan).
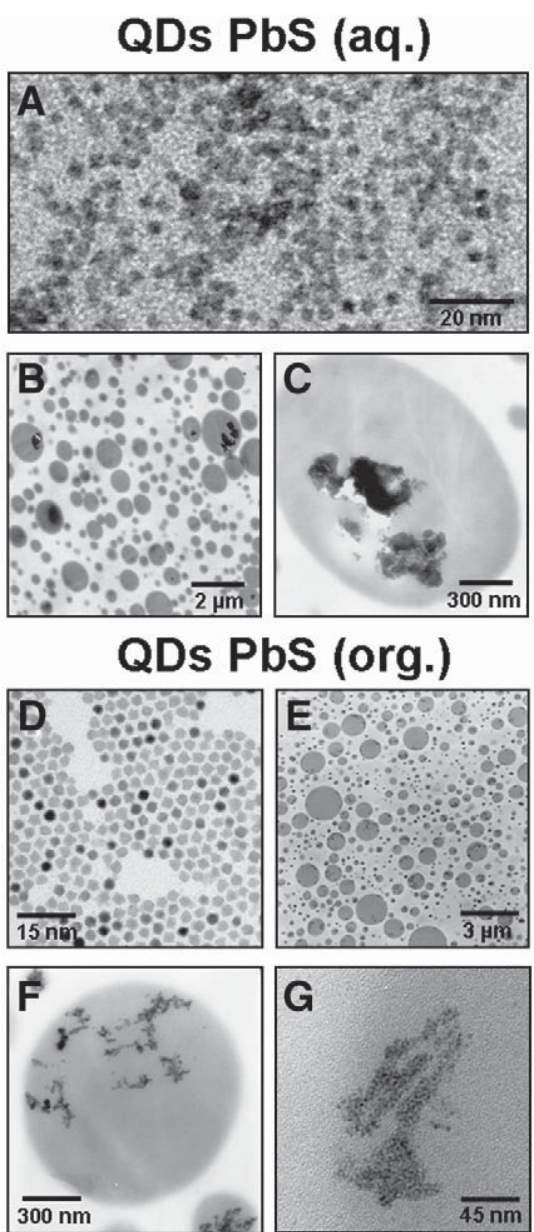

Fig. 1. Transmission electron microscopy (TEM) images of PbS nanocrystals (NCs) before and after encapsulation into PLGA microspheres (PLGA-MS). (A) Hydrophilic PbS NCs (QDs PbS (aq.)) directly after synthesis. (B,C) TEM images of PLGA-MS preparations after including $\mathrm{PbS}$ (aq.) into the aqueous phase of the spray drying emulsion. (D) Hydrophobic PbS NCs (ODs PbS (org.)) directly after synthesis. (E,F) TEM images of PLGA-MS preparations after including QDs $\mathrm{PbS}$ (org.) into the organic phase of the spray drying emulsion. (G) Image of encapsulated QDs PbS (org.) within a PLGA MS at higher magnification. Scale bars indicate the size of the images. PLGA-MS images were performed from $50 \mathrm{~nm}$ slices. Figure panels show representative images.
2.8. Magnetic depletion of phagocytes by PLGA-MS containing superparamagnetic iron oxide nanoparticles (SPIONs)

PLGA-MS containing SPIONs were prepared as described above. $50 \mu \mathrm{g} / \mathrm{ml}$ magnetic or empty PLGA-MS were added to heterogeneous 50:50 cell suspensions of B8 $\left(\mathrm{H}-2^{\mathrm{d}}\right)$ and DC2.4 $\left(\mathrm{H}-2^{\mathrm{b}}\right)$ cells for $1 \mathrm{~h}$. For magnetic separation of phagocytes, cells were applied to MACS columns (Miltenyi Biotec) and washed with phosphate buffered saline. Specific depletion of phagocytes was analysed by flow cytometry using FITC anti-mouse $\mathrm{H}-2 \mathrm{D}^{\mathrm{b}}$ and PE anti-mouse $\mathrm{H}-2 \mathrm{D}^{\mathrm{d}}$ (BD Biosciences). Specific depletion was calculated following this equation: specific depletion $=(1-[$ ratio non-sorted $/$ ratio sorted $] \times 100$, and ratio $=\% \mathrm{H}-$ $2 \mathrm{D}^{\mathrm{b}+} / \% \mathrm{H}-2 \mathrm{D}^{\mathrm{d}+}$.

\section{Results}

3.1. Efficient encapsulation of $\mathrm{PbS}$ quantum dots after addition of nanoparticles to the organic phase of the spray drying process

The encapsulation of QDs into PLGA-MS can be achieved by two possible strategies. The PLGA emulsion used for spray drying consists of an organic and an aqueous phase. NCs to be encapsulated can therefore be added to either one of the two phases. In order to test both possibilities, we first tried to encapsulate NCs via the aqueous phase of the emulsion. Therefore, PbS QDs were hydrophilised by exchanging the stabilizing hydrophobic ligand against a hydrophilic one (PbS QDs (aq.)). Using these particles, we achieved encapsulation of electron dense material into PLGA-MS (Fig. 1B,C). However the QD inclusions were not evenly distributed throughout the microspheres and only low numbers of particles showed QD incorporation. At the same time, PLGA-MS that did contain QDs were found to contain large aggregates of nanoparticles. In a second experimental setting we used hydrophobic QDs (PbS QDs (org.)) to reach our aim. Indeed, addition of PbS QDs (org.) into the organic phase of the spray drying emulsion led to an improved distribution of NCs inclusions (Fig. 1E, F). PLGA-MS were prepared for TEM analysis and were found to be entirely labelled with electron dense NCs. The distribution of QDs within single microspheres was smoother, compared to the preparation in aqueous solution (Fig. 1G).

A comparison of the two experimental strategies revealed that the addition of QDs into the organic phase of the spray drying emulsion led to the most efficient and even encapsulation of nanoparticles into the PLGA-MS. Hence, this experimental setup was chosen for all further experiments.

3.2. Encapsulation of PbS quantum dots is a suitable methods to label PLGA microspheres with an electron dense marker for electron microscopy

Initially we started to encapsulate QDs into PLGA-MS to have an electron dense marker that allows identification of microspheres after phagocytotic uptake by immune cells. The labelling of PLGA-MS with a defined electron dense material is essential to distinguish phagocytosed microparticles from subcellular structures that also occur in the absence of microspheres (Fig. 2, control). As shown in Fig. 1, we successfully labelled PLGA-MS with electron dense PbS QDs and were therefore able to identify PLGA-MS within the cytoplasm of bone marrow-derived dendritic cells (BMDCs), when analyzing TEM images of samples after phagocytosis. Interestingly, we found most microspheres present in larger vacuole-like organelles, each containing multiple particles. These structures were observed at 4 and $48 \mathrm{~h}$ after addition of labelled microspheres to BMDCs (Fig. 2; upper right, lower left).

As we were interested whether microspheres after phagocytotic uptake do enter the cytoplasm or stay in defined membrane-enclosed compartments, we had a closer look at cellular positions, where 
control

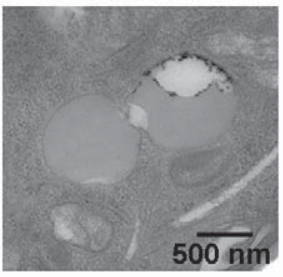

$48 \mathrm{~h}$

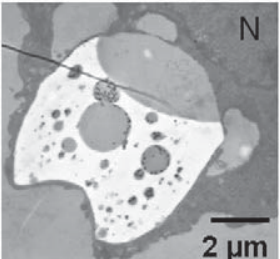

$2 \mu \mathrm{m}$ control

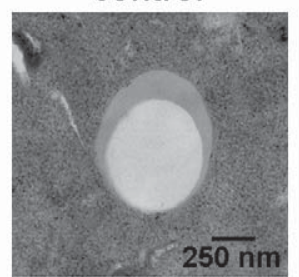

$48 \mathrm{~h}$

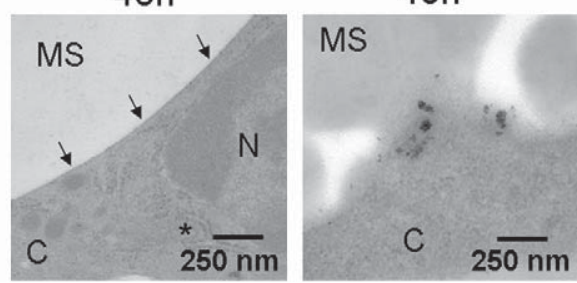

Fig. 2. Transmission electron microscopy (TEM) images of PbS quantum dot-labelled PLGA microspheres after uptake by bone marrow derived dendritic cells (BMDCs). BMDCs were grown on petriPERM $₫$ dishes and microspheres were added or not (control). After $1 \mathrm{~h}$ extracellular microspheres were washed off and BMDCs were further inoculated in medium for the indicated time points before preparation for TEM. The following abbreviations are used: MS, microsphere; N, nucleus; *, endoplasmatic reticulum with ribosomes; C, cytoplasm. Scale bars indicate the size of cellular structures. Arrows indicate enclosing membrane. Images were obtained from $50 \mathrm{~nm}$ slices. Figure shows representative images.

microspheres and cytoplasm are in close proximity (Fig. 2; lower middle, lower right). For some contact sides it was evident that microspheres and cytoplasm were separated by a surrounding membrane. Other slices depicted microspheres that fused with the cytoplasm without a visible membrane. We observed microspheres that were surrounded by a membrane $48 \mathrm{~h}$ after particle uptake. However, we wanted to confirm these results with an independent method. Therefore we performed uptake experiments with fluorescent PLGA-MS, followed by confocal LSM.

3.3. Encapsulation of fluorescent CdSe QDs shows co-localization of PLGA-MS with LAMP1 ${ }^{+}$organelles in a dendritic cell line even $72 \mathrm{~h}$ after phagocytosis

After having established the encapsulation of PbS QDs into the PLGA-MS preparations, we applied this procedure to NCs with other properties. Here we used CdSe QDs with an emission maximum of $583 \mathrm{~nm}$ to study intracellular distribution of PLGA-MS by confocal LSM. Encapsulation was performed by adding nanoparticles to the organic phase of the spray drying emulsion. Indeed, fluorescent CdSe QDs were efficiently encapsulated into the PLGA-MS (TEM, data not shown).

Material which is engulfed by phagocytes usually ends up in the late endosomal/lysosomal compartment of the cell, where digestion is enabled by acidification [26]. In order to investigate whether microspheres are targeted to the lysosomal compartment, we chose LAMP1 as a marker molecule to label the membrane of lysosomes. At different time points after phagocytosis of fluorescent PLGA-MS by the DCs line DC2.4, we looked for co-localisation with the lysosomal marker LAMP1 (Fig. 3). Shortly after addition of PLGA-MS (10 min) we observed attachment of particles to the outer membrane of the cells. Microspheres were not labelled by anti-LAMP1 staining (green). After 30 min microspheres were already taken up by DC2.4 cells. However, no co-localization with LAMP1 could be observed at this early time point. Interestingly, as early as $2 \mathrm{~h}$ after addition of microspheres, $\mathrm{LAMP}^{+}$staining appeared around the microparticles, indicating the presence of a lysosomal membrane. This co-localization was getting even more prominent with time. At all later time points analysed, microspheres were surrounded by a distinct LAMP1 ${ }^{+}$staining $(24 \mathrm{~h}$ and $48 \mathrm{~h}$, data not shown). From these data we conclude that the microspheres were efficiently taken up by DC2.4 cells and enter the lysosomal compartment as early as $2 \mathrm{~h}$ after phagocytosis. Within the first $72 \mathrm{~h}$ microspheres did not enter the cytoplasm, but localized in LAMP1 $^{+}$organelles.

3.4. Microspheres taken up by different primary antigen presenting cells localize in LAMP1 ${ }^{+}$organelles even $48 \mathrm{~h}$ after phagocytosis

In order to extend the data observed for the cell line DC2.4, we prepared primary mouse DCs and macrophages, to further examine the intracellular fate of phagocytosed microparticles. Interestingly, also for these primary cells it was evident that both, at $4 \mathrm{~h}$ and $48 \mathrm{~h}$ after phagocytosis, microspheres were surrounded by a bright LAMP1 $^{+}$staining (Fig. 4), indicating the presence of a lysosomal membrane. For both cell types and time points we were not able to find significant numbers of microspheres that were not enclosed by bright LAMP1 staining. Hence, we conclude, that the lysosomal storage of PLGA-MS within LAMP1 ${ }^{+}$organelles is a general feature that is common in all APC lines analysed. This lysosomal storage has direct implications for the understanding of antigen presentation following microsphere based vaccination.

\subsection{Efficient cross-presentation of PLGA MS-encapsulated ovalbumin by} APCs despite of lysosomal storage of microparticles

The lysosomal storage of PLGA-MS is a crucial parameter for the cross-presentation pathway. For the activation of $\mathrm{CD}^{+} \mathrm{T}$-cells by vaccination, it is critical that antigenic peptides are presented on MHC class I molecules [27]. As discussed later, this cross-presentation of PLGA-MS-encapsulated antigens was shown to be dependent on proteins entering the "direct presentation pathway". Our observation of a lysosomal storage of PLGA-particles argues that cross-presentation can only occur after translocation of proteins, but not entire particles, from the endo/lysosomal compartment into the cytosol. We analysed the intracellular fate of PLGA-MS in three different cell types. Performing an in vitro assay, we wanted to demonstrate that cross-presentation of encapsulated ovalbumin can be observed in these cells, despite of lysosomal localization of PLGA particles. DC2.4 cells, BMDCs, and pMФs were incubated with MS containing either ovalbumin (MS OVA) or left empty as control (MS empty). Additionally, $\mathrm{OVA}_{257-264}$ specific $\mathrm{CD}^{+}$ T-cell hybridomas were added to detect cross-presentation of the SIINFEKL epitope on MHC class I molecules. As demonstrated for other APCs, all three cell types were able to efficiently cross-present MS-encapsulated OVA after $16 \mathrm{~h}$ (Fig. 5). From this finding we conclude that antigens encapsulated into PLGA-MS are released from hydrolysing particles within the endo/lysosomal compartment and are then translocated into the cytoplasm.

3.6. Encapsulation of super-paramagnetic iron oxide nanoparticles (SPIONs) into PLGA-MS for depletion of phagocytes from biological samples

PLGA-MS in the micrometer range are efficiently taken up by phagocytes but not other cell types. This specific difference can be utilized to deplete phagocytes from biological samples by magnetic sorting using encapsulated SPIONs. In a straight forward proof of principle experiment we tried to deplete dendritic cells from a heterogeneous cell culture. The murine fibroblast cell lines B8 (BALB/ c-derived, $\mathrm{H}-2^{\mathrm{d}}$ ) and the DC cell line DC2.4 (C57BL/6-derived, $\mathrm{H}-2^{\mathrm{b}}$ ) were cultured in a $\sim 1: 1$ ratio. To illustrate the possibility to magnetically deplete DCs, PLGA-MS containing SPIONs (MS-SPIONs) or empty microspheres (MS-empty) were added to the cell mixture for $1 \mathrm{~h}$. Afterwards cells were harvested and applied to a magnetic column. The two cell lines can be distinguished due to differences in the expression of the major histocompatibility complex (MHC) class I alleles ( $\mathrm{H}-2^{\mathrm{b}}$ and 

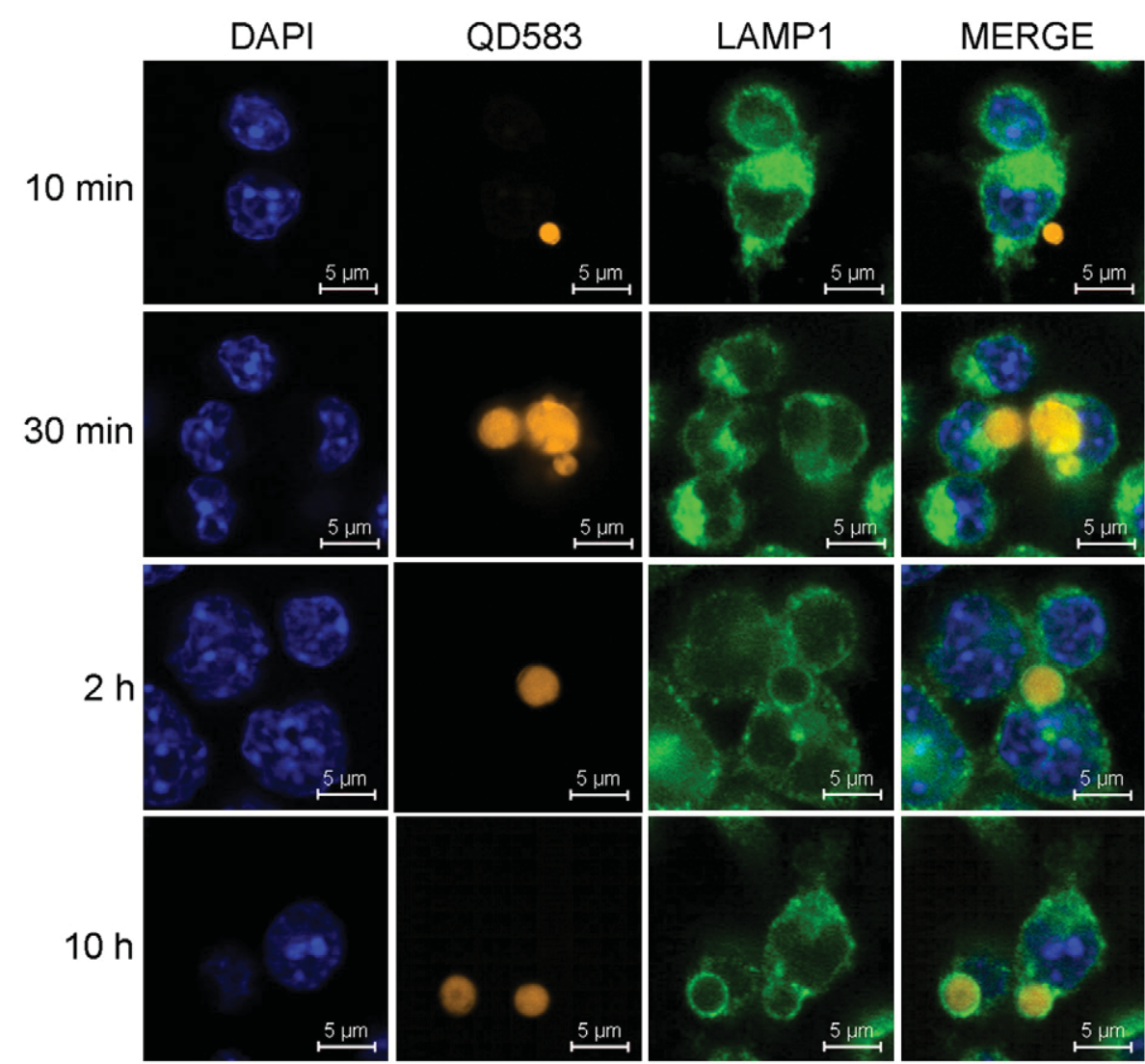

$72 \mathrm{~h}$
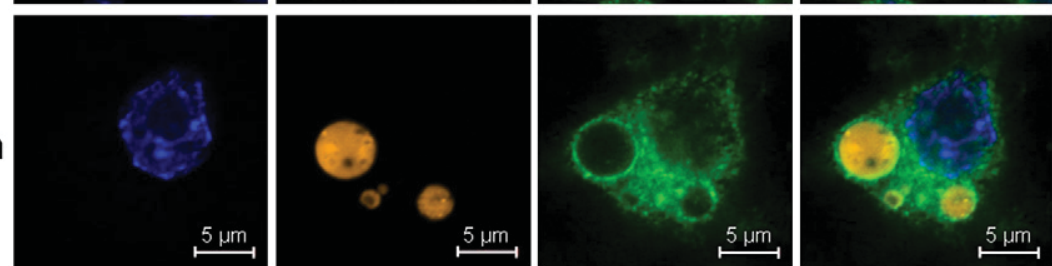

Fig. 3. Confocal images showing the uptake of fluorescent microspheres by DC2.4 cells and co-localisation with the lysosomal marker LAMP1. DC2.4 cells were grown on cover slips and fluorescent CdSe quantum dot-labelled microspheres were added. After $1 \mathrm{~h}$ (10 and $30 \mathrm{~min}$ for the first two time points) unbound microspheres were removed by washing with phosphate buffered saline and cells were further incubated in medium. At indicated time points cover slips were washed with phosphate buffered saline and cells were fixed in $4 \%$ paraformaldehyde. Confocal laser scanning microscopy was performed after intracellular staining of cell nuclei (DAPI, blue) and the lysosomal marker LAMP1 (LAMP1, green). CdSe quantum dot-labelled microspheres were excited with a UV-laser (QD583, yellow). Images in the very right panel show merged channels (MERGE). Shown examples are representative images from the indicated time points after co-incubation.

$\left.H-2^{d}\right)$. The flow through was collected and analysed for the expression of $\mathrm{H}-2$ molecules (Fig. 6). We were able to magnetically deplete around $90 \%$ of DC2.4 cells from the heterogeneous culture indicating the usefulness of this approach.

\section{Discussion}

Attempts to combine the properties of NCs with biocompatible polymer particles are still restricted to very few examples. In two recent publications, a method was introduced to externally label PLGA nanoparticles with NCs as potential biological probe for bioimaging and MRI spectroscopy [28,29]. The authors show homogenous distribution of NCs covering PLGA particle surface by TEM and are able to detect fluorescent PLGA nanoparticles by fluorescence microscopy. Other efforts illustrate the possibility to encapsulate NCs into PLGA particles using the nanoprecipitation method $[30,31]$. In this report, we introduce a novel protocol to efficiently encapsulate NCs into PLGA-MS using the spray drying method. Encapsulation by spray drying has a number of advantages. First, it does not modify the surface characteristics of PLGA particles, which might alter their characteristic biodistribution pattern. Second, generation of NC-encapsulated PLGA-MS by spray drying does not require additional purification of particles from nonencapsulated NCs, as needed for nanoprecipitation [30]. The encapsulation of NCs described here can be easily performed by any laboratory using the spray drying technique to produce PLGAMS, without any procedural adaptation.

The results presented in this report are to our knowledge the first examples to illustrate the potential of PLGA-encapsulated NCs as a tool to study intracellular distribution of particles by TEM and confocal co-localization studies. Understanding the intracellular fate of particulate vaccines after uptake by APCs is critical for the development of successful immunotherapies. For the induction of protective cytotoxic T-cell responses against cancer or other tissue specific immune targets, it is critical that the antigen encapsulated into PLGA-MS is targeted to the MHC class I presentation machinery of APCs. Only these specialised immune cells are able to initially activate naïve T-cells by providing co-stimulatory signals [32]. Generally, there are two pathways known on how antigen-derived peptides can be presented on MHC class I molecules. Endogenous proteins that are synthesized within the cell are usually degraded via the ubiquitin/ proteasome system into polypeptides that are loaded onto MHC I molecules within the endoplasmatic reticulum [33]. From there $\mathrm{MHC}$ /peptide complexes are translocated to the cell surface to allow 

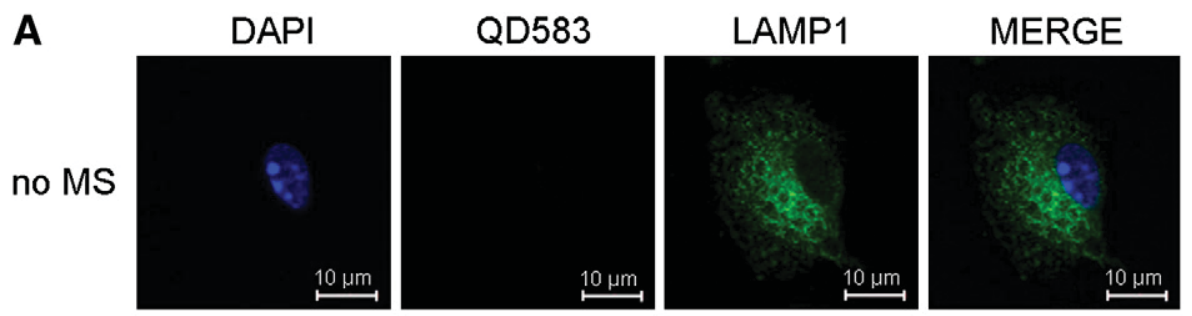

$4 \mathrm{~h}$
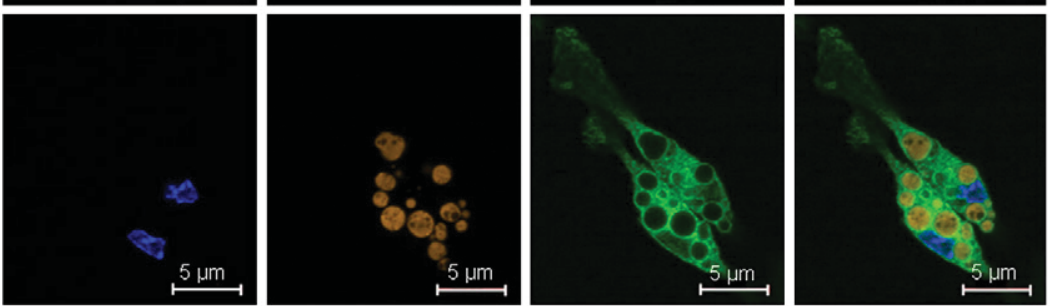

$48 \mathrm{~h}$
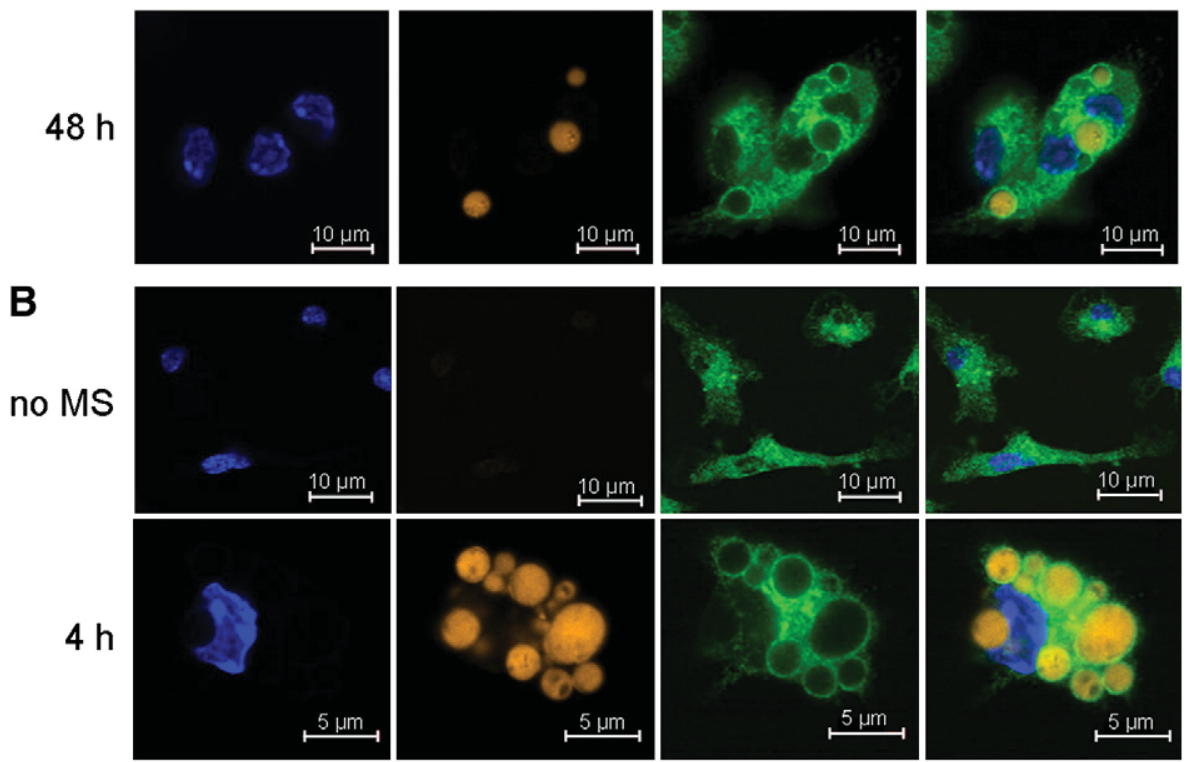

$48 \mathrm{~h}$
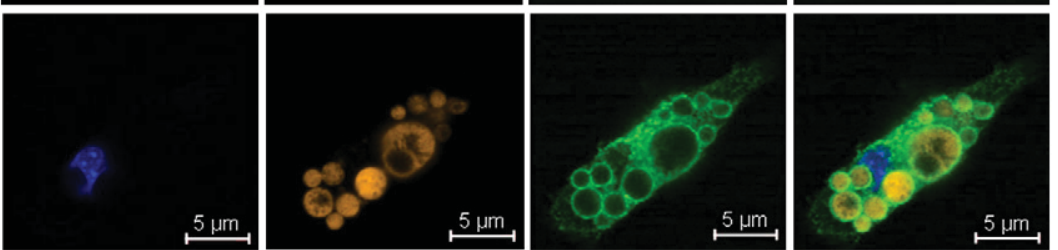

Fig. 4. Confocal laser scanning microscopy (LSM) images of fluorescent CdSe quantum dot-labelled microspheres after uptake by primary peritoneal macrophages (A) and bone marrow derived dendritic cells (BMDCs) (B) and co-staining for the lysosomal marker LAMP1. Cells were grown on cover slips and fluorescent CdSe quantum dot-labelled microspheres were added for $1 \mathrm{~h}$. Unbound microspheres were washed off with phosphate buffered saline and cells were further incubated in medium. At indicated time points cells were fixed with $4 \%$ paraformaldehyde and intracellular staining of cell nuclei (DAPI, blue) and the lysosomal marker LAMP1 (LAMP1, green) was performed. CdSe quantum dot-labelled microspheres were excited with the UV-laser (QD583, yellow). Images on the very right show merged channels (MERGE). Shown examples are representative results for the indicated time points.

T-cell receptor signalling. This way of MHC class I presentation is referred to as the "direct-presentation pathway". In contrast, proteins that are taken up by APCs from extracellular compartments primarily enter the endo/lysosomal pathway. However, peptides derived from such proteins can still be presented on MHC class I via a pathway termed "cross-presentation" [27]. For the cross-presentation of antigens that are encapsulated into PLGA-MS the intracellular localization of hydrolyzing particles is critical to understand the mode of action of this vaccine. There are several publications addressing the intracellular localization after administration of PLGA particles. However, the data are inconsistent and two hypotheses can be found. In one of the first studies which analysed the intracellular distribution of biodegradable particles the authors used colloidal gold labelling to visualize nanoparticles $(40-120 \mathrm{~nm}$ ) by TEM [1]. In this study particles were found to enter the cytoplasm and could additionally be detected in Golgi and secretory vesicles of liver and epithelium cell. This paper was followed by a number of other publications that independently showed the endo/lysosomal escape of PLGA particles after uptake by МФs [15], smooth muscle cells [11], and epithelial cells [12]. Biochemical evidence for an endosomal escape was provided by studying DCs and B-cells [10]. An endosomal escape would allow release of encapsulated proteins into the cytoplasm, where they could be targeted to the "direct-presentation" machinery. However, a number of other publications using similar techniques were published, finding no evidence for cytosolic transition of PLGA particles. A study using DCs and MФs showed the presence of a phagocytotic membrane after uptake of particles [2]. Independent reports showed membrane-enclosed PLGA particles after uptake by peritoneal exudates cells [13] and peritoneal MФs [16]. These findings would favour other mechanisms for the cross- 


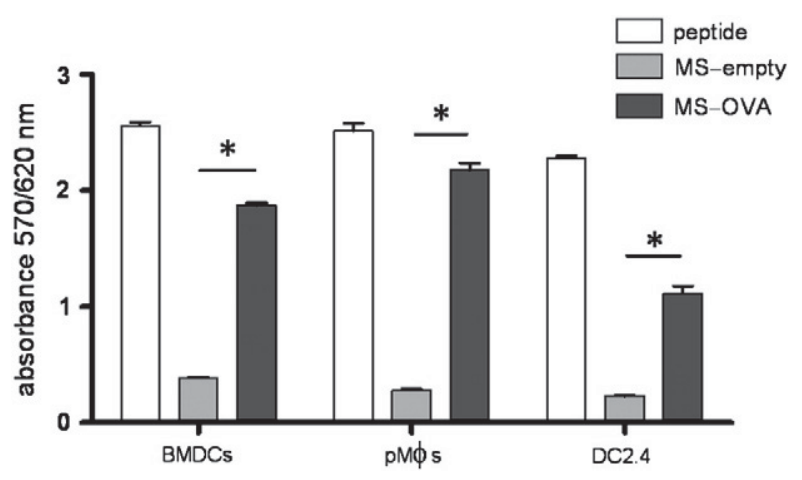

Fig. 5. In vitro cross-presentation of PLGA-MS-encapsulated ovalbumin by different antigen presenting cells $16 \mathrm{~h}$ after uptake. DC2.4 cells, bone marrow-derived dendritic cells (BMDCs), or peritoneal macrophages (pMФs) were incubated with $\mathrm{OVA}_{257-264}$ specific $\mathrm{CD}^{+}{ }^{+} \mathrm{T}$-cell hybridomas $\mathrm{B} 3 \mathrm{Z}$, in the presence of either external $\mathrm{OVA}_{257-264}$ peptide (peptide), empty microspheres (MS-empty) or microspheres containing OVA (MS-OVA). Activation of B3Z cells was detected in a colorimetric LacZ assay (absorbance at $570 \mathrm{~nm}$ with reference wave length of $620 \mathrm{~nm}$ ). Significance was calculated by student's t-test from two independent experiments: $\left({ }^{*}\right)=\mathrm{p}<0.05$.

presentation of encapsulated antigen. Nevertheless, there is a general consensus that either the encapsulated antigens or the entire PLGA particles have to enter the cytoplasm in order to achieve efficient cross-presentation [34]. The heterogeneous results observed for this important question, may be in part due to differences in cell-types
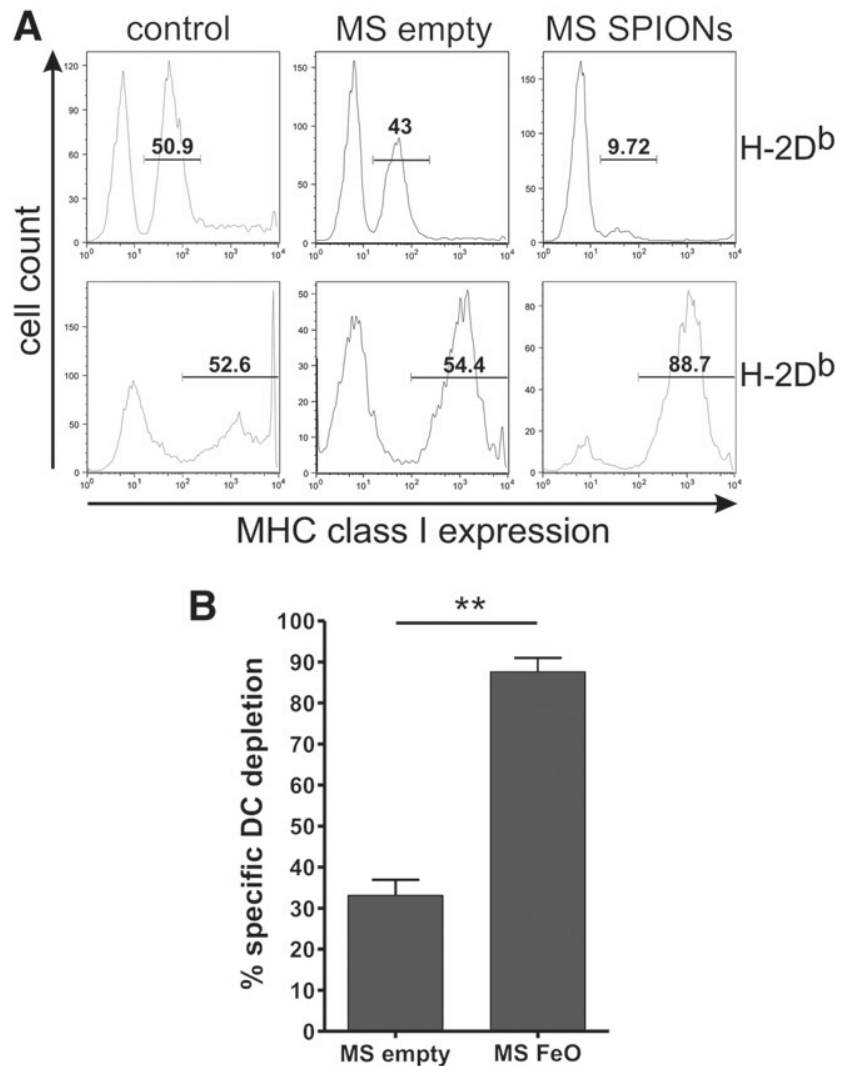

Fig. 6. In vitro depletion of dendritic cells from a heterogeneous culture. The murine fibroblast cell line B8 $\left(\mathrm{H}-2^{\mathrm{b}}\right)$ and the dendritic cell line DC2.4 $\left(\mathrm{H}-2^{\mathrm{d}}\right)$ were cultured over night in a ratio of $1: 1$. For depletion of phagocytes, PLGA-MS containing either superparamagnetic iron oxide nanoparticles (MS SPIONs) or left empty as control (MS empty) were added to the cell culture for $1 \mathrm{~h}$. Afterwards, cells were harvested and applied to a magnetic column. (A) The flow through was collected and cells were analysed for the expression of $\mathrm{H}-2 \mathrm{D}^{\mathrm{b} / \mathrm{d}}$ by flow cytometry. (B) Specific depletion of DC2.4 cells was calculated as described in the methods section. Significance was calculated by student's t-test from two independent experiments: $\left(^{* *}\right)=\mathrm{p}<0.01$. used, protocols for PLGA polymerization and size distribution of particles. However, also the lack of electron dense material in PLGA preparations for TEM and photobleaching of conventional dyes might be responsible for misinterpretations. To circumvent this, we established a protocol to label PLGA-MS with NCs. Our TEM analyses not only showed the efficient encapsulation of NCs, but also indicate the presence of an enclosing membrane $48 \mathrm{~h}$ after phagocytotic uptake of PLGA particles. More evidence came from our experiments performed by confocal LSM. Fluorescent QDs were encapsulated into PLGA-MS and allowed co-localisation of intracellular particles with the lysosomal marker LAMP1. It was evident that even after $48 \mathrm{~h}$ PLGA-MS were located inside of LAMP1 ${ }^{+}$organelles. The kinetic of particle uptake confirmed earlier studies performed by TEM [13]. Taken together, our data show the lysosomal storage of PLGA-MS after phagocytosis (Figs. 2-4). This has direct implication for the crosspresentation of encapsulated antigen, since MHC class I presentation already occurs at much earlier time points (Fig. 5). We hypothesise that not the entire PLGA particles, but proteins released within the lysosomes do cross the lysosomal membrane to enter the direct MHC class I loading machinery.

In the third part, we showed that encapsulation of SPIONs into PLGA-MS leads to magnetic particles that can be applied for magnetic cell sorting. Magnetic nanoparticles are studied as contrast agents for magnetic resonance imaging (MRI) in humans [35]. The small size of particles allows distribution with the blood stream throughout the organism. SPIONs itself are not toxic and can be applied in vivo [36]. To specifically label malignant tissues there were attempts to couple SPIONs to tumor-specific antibodies [37]. This approach would allow exact determination of tumor size and distribution by MRI. Here we introduce the encapsulation of magnetic nanoparticles into PLGA-MS using the spray drying method. PLGA-MS in the micrometer range are specifically endocytosed by phagocytes only. Therefore, we were able to successfully use magnetic PLGA-MS to clear a biological sample from phagocytic cells. This technology is specific for phagocytes but independent of antibody recognition. It might therefore be an easy and straightforward way to deplete phagocytes from a mixed suspension of cells.

\section{Conclusions}

Here we report on a method on how PLGA-MS, successfully used as antigen carrier devices in vaccination, can be labelled either with electron dense PbS nanocrystals for intracellular localization of MS by electron microscopy or with CdSe nanocrystals for fluorescence microscopy. Microencapsulation of nanocrystals into PLGA-MS by spray drying allowed us to localize PLGA-MS within dendritic cells and macrophages at different time points after phagocytosis. We show that PLGA-MS do not escape endosomes but remain in LAMP1+ lysosomes up to three days after uptake although cross-presentation of microencapsulated antigen occurred much earlier. Microencapsulated nanocrystals are therefore instrumental for elucidating the cell biological pathways of the extraordinarily efficient cross-presentation afforded by PLGA-MS. Furthermore, we show that ferromagnetic nanocrystals can be used for the efficient magnetic separation of phagocytic and non-phagocytic cells.

\section{Acknowledgements}

We thank Elisa May and the Bioimaging Center of Konstanz University for experimental support in fluorescence microscopy. We acknowledge the contribution of the DC2.4 cell line by Kenneth Rock and the B3Z hybridoma by Dr. Nilabh Shastri. This study was supported by a grant from Deutsche Krebshilfe (grant no. 107943 to M.G.). The authors declare that they have no conflict of interest. 


\section{References}

[1] E. Mathiowitz, J.S. Jacob, Y.S. Jong, G.P. Carino, D.E. Chickering, P. Chaturvedi, C.A. Santos, K. Vijayaraghavan, S. Montgomery, M. Bassett, C. Morrell, Biologically erodable microspheres as potential oral drug delivery systems, Nature 386 (1997) 410-414.

[2] E. Walter, D. Dreher, M. Kok, L. Thiele, S.G. Kiama, P. Gehr, H.P. Merkle, Hydrophilic poly(DL-lactide-co-glycolide) microspheres for the delivery of DNA to humanderived macrophages and dendritic cells, J. Control. Release 76 (2001) 149-168.

[3] Y. Waeckerle-Men, B. Gander, M. Groettrup, Delivery of tumor antigens to dendritic cells using biodegradable microspheres, Methods Mol. Med. 109 (2005) 35-46.

[4] E. Schlosser, M. Mueller, S. Fischer, S. Basta, D.H. Busch, B. Gander, M. Groettrup, TLR ligands and antigen need to be coencapsulated into the same biodegradable microsphere for the generation of potent cytotoxic T lymphocyte responses, Vaccine 26 (2008) 1626-1637.

[5] X. Peng, An essay on synthetic chemistry of colloidal nanocrystals, Nano Res. 2 (2009) 425-447.

[6] X. Gao, L. Yang, J.A. Petros, F.F. Marshall, J.W. Simons, S. Nie, In vivo molecular and cellular imaging with quantum dots, Curr. Opin. Biotechnol. 16 (2005) 63-72.

[7] U.I. Tromsdorf, N.C. Bigall, M.G. Kaul, O.T. Bruns, M.S. Nikolic, B. Mollwitz, R.A. Sperling, R. Reimer, H. Hohenberg, W.J. Parak, S. Forster, U. Beisiegel, G. Adam, H. Weller, Size and surface effects on the MRI relaxivity of manganese ferrite nanoparticle contrast agents, Nano Lett. 7 (2007) 2422-2427.

[8] C.D.M. Doneg, P. Liljeroth, D. Vanmaekelbergh, Physicochemical evaluation of the hot-injection method, a synthesis route for monodisperse nanocrystals, Small 12 (2005) 1152-1162.

[9] K. Cal, K. Sollohub, Spray drying technique. I: hardware and process parameters, J. Pharm. Sci. 99 (2010) 575-586.

[10] H. Shen, A.L. Ackerman, V. Cody, A. Giodini, E.R. Hinson, P. Cresswell, R.L. Edelson, W.M. Saltzman, D.J. Hanlon, Enhanced and prolonged cross-presentation following endosomal escape of exogenous antigens encapsulated in biodegradable nanoparticles, Immunology 117 (2006) 78-88.

[11] J. Panyam, W.Z. Zhou, S. Prabha, S.K. Sahoo, V. Labhasetwar, Rapid endo-lysosomal escape of poly(DL-lactide-co-glycolide) nanoparticles: implications for drug and gene delivery, FASEB J. 16 (2002) 1217-1226.

[12] M.S. Cartiera, K.M. Johnson, V. Rajendran, M.J. Caplan, W.M. Saltzman, The uptake and intracellular fate of PLGA nanoparticles in epithelial cells, Biomaterials 30 (2009) 2790-2798.

[13] A. de Jesus Gomes, C.N. Lunardi, F.H. Caetano, L.O. Lunardi, A.E. da Hora Machado, Phagocytosis of PLGA microparticles in rat peritoneal exudate cells: a timedependent study, Microsc. Microanal. 12 (2006) 399-405.

[14] M. Yoshida, J.E. Babensee, Molecular aspects of microparticle phagocytosis by dendritic cells, J. Biomater. Sci. Polym. Ed. 17 (2006) 893-907.

[15] A.J. Gomes, A.S. Faustino, A.E. Machado, M.E. Zaniquelli, T. de Paula Rigoletto, C.N. Lunardi, L.O. Lunardi, Characterization of PLGA microparticles as a drug carrier for 3-ethoxycarbonyl-2 h-benzofuro[3, 2-f]-1-benzopyran-2-one. Ultrastructural study of cellular uptake and intracellular distribution, Drug Deliv. 13 (2006) 447-454.

[16] A.P. Trombone, C.L. Silva, L.P. Almeida, R.S. Rosada, K.M. Lima, C. Oliver, M.C. Jamur, A.A. Coelho-Castelo, Tissue distribution of DNA-Hsp65/TDM-loaded PLGA microspheres and uptake by phagocytic cells, Genet. Vaccines Ther. 5 (2007) 9.

[17] M. Peyre, R. Fleck, D. Hockley, B. Gander, D. Sesardic, In vivo uptake of an experimental microencapsulated diphtheria vaccine following sub-cutaneous immunisation, Vaccine 22 (2004) 2430-2437.
[18] S. Miltenyi, W. Muller, W. Weichel, A. Radbruch, High gradient magnetic cell separation with MACS, Cytometry 11 (1990) 231-238.

[19] A. Grutzkau, A. Radbruch, Small but mighty: how the MACS-technology based on nanosized superparamagnetic particles has helped to analyze the immune system within the last 20 years, Cytom. A 77 (2010) 643-647.

[20] M. Nagel, S.G. Hickey, A. Fromsdorf, A. Kornowski, H. Weller, Synthesis of monodisperse $\mathrm{PbS}$ nanoparticles and their assembly into highly ordered 3D colloidal crystals, Z. Phys. Chem. 221 (2007) 427-437.

[21] I. Mekis, D.V. Talapin, A. Kornowski, M. Haase, H. Weller, One-pot synthesis of highly luminescent $\mathrm{CdSe} / \mathrm{CdS}$ core-shell nanocrystals via organometallic and "greener" chemical approaches, J. Phys. Chem. B 107 (2003) 7454-7462.

[22] D.V. Talapin, I. Mekis, S. Gotzinger, A. Kornowski, O. Benson, H. Weller, CdSe/CdS/ $\mathrm{ZnS}$ and CdSe/ZnSe/ZnS core-shell-shell nanocrystals, J. Phys. Chem. B 108 (2004) 18826-18831.

[23] A.R. Spurr, A low-viscosity epoxy resin embedding medium for electron microscopy, J. Ultrastruct. Res. 26 (1969) 31-43.

[24] J. Karttunen, S. Sanderson, N. Shastri, Detection of rare antigen-presenting cells by the lacZ T-cellactivation assay suggests an expression cloning strategy for T-cell antigens, Proc. Natl Acad. Sci. USA 89 (1992) 6020-6024.

[25] M. Groettrup, T. Ruppert, L. Kuehn, M. Seeger, S. Standera, U. Koszinowski, P.M. Kloetzel, The interferon- $\gamma$-inducible $11 \mathrm{~S}$ regulator (PA28) and the LMP2/LMP7 subunits govern the peptide production by the $20 \mathrm{~S}$ proteasome in vitro, J. Biol. Chem. 270 (1995) 23808-23815.

[26] K. Honey, A.Y. Rudensky, Lysosomal cysteine proteases regulate antigen presentation, Nat. Rev. Immunol. 3 (2003) 472-482.

[27] C. Kurts, B.W.S. Robinson, P.A. Knolle, Cross-priming in health and disease, Nat. Rev. Immunol. 10 (2010) 403-414.

[28] F.Y.Cheng, S.P. Wang, C.H. Su, T.L. Tsai, P.C. Wu, D.B. Shieh, J.H. Chen, P.C. Hsieh, C.S Yeh, Stabilizer-free poly(lactide-co-glycolide) nanoparticles for multimodal biomedical probes, Biomaterials 29 (2008) 2104-2112.

[29] W.S. Kuo, Stabilizer-free poly(lactide-co-glycolide) nanoparticles conjugated with quantum dots as a potential carrier applied in human mesenchymal stem cells, J. Chin. Chem. Soc. 56 (2009) 940-948.

[30] B.J. Nehilla, P.G. Allen, T.A. Desai, Surfactant-free, drug-quantum-dot coloaded poly(lactide-co-glycolide) nanoparticles: towards multifunctional nanoparticles, ACS Nano 2 (2008) 538-544.

[31] C.-H. Yang, K.-C. Lin, Y.-H. Chang, Y.-C. Lin, Quantum dots CdSe/ZnS-loaded Poly (D,L-lactide-co-glycolide) nanoparticles: physicochemical characterization and application, Mater. Sci. Forum 505-507 (2006) 667-672.

[32] I. Mellman, R.M. Steinman, Dendritic cells: specialized and regulated antigen processing machines, Cell 106 (2001) 255-258.

[33] M. Groettrup, M. van den Broek, K. Schwarz, A. Macagno, S. Khan, R. de Giuli, G Schmidtke, Structural plasticity of the proteasome and its function in antigen processing, Crit. Rev. Immunol. 21 (2001) 339-359.

[34] R. Audran, K. Peter, J. Dannull, Y. Men, M. Groettrup, B. Gander, G. Corradin, Encapsulation of peptides prolongs their presentation to cytotoxic $\mathrm{T}$ cells by antigen presenting cells in vitro, Vaccine 21 (2003) 1250-1255.

[35] O. Pankhurst, J. Connolly, S. Jones, J. Dobson, Applications of magnetic nanoparticles in biomedicine, J. Phys. D Appl. Phys. 36 (2003) R167-R181.

[36] R. Hergt, S. Dutz, R. Muller, M. Zeisberger, Magnetic particle hyperthermia: nanoparticle magnetism and materials development for cancer therapy, J. Phys. Condens. Matter 18 (2006) S2919-S2934.

[37] J. Yang C.-H. Lee, J. Park, S. Seo, E.-K. Lim, YJ. Song J.-S. Suh, H.-G. Yoon, Y.-M. Huh, S. Haam, Antibody conjugated magnetic PLGA nanoparticles for diagnosis and treatment of breast cancer, J. Mater. Chem. 17 (2007) 2695-2699. 